\title{
A Comparative Study of Swarm Intelligence-Based Optimization Algorithms in WSN
}

\author{
Aya Ayad Hussein ${ }^{1}$ and Rajaaaldeen Abd Khalid ${ }^{2}$ \\ ${ }^{1 \& 2}$ College of Information Engineering, Al-Nahrain University, Baghdad, Iraq \\ E-Mail: aya.ayad.it@gmail.com, rarajaaalden9@gmail.com
}

\begin{abstract}
In the last decades, WSN gets all the attention in research and applications especially in science and engineering fields due to it is great benefits which been introduced. These networks are used in rough and inaccessible environments such as battlefields, volcanoes, and forests, so basically there is a low chance to recharge or change the low battery or dead nodes. Hence, WSNs are hypersensitive and vulnerable to energy more than other classic wireless networks. Therefore, this led to emerging improvements rapidly in WSN to reach the goal of achieving the network requirements and satisfying the user needs at the same time to get the best results. Artificial Intelligent systems Toke the biggest share of the WSN development process. So, In this paper we will focus on an important section in an intelligent system based on optimization algorithms, is the swarm intelligence that depends on the real behavior of animals and insects colonies, it is a system that based on many individuals that working within a group as a team and coordinate their behavior using decentralized control and self- organization. SI-based optimization algorithms have a great and affirmative influence on WSN represented by minimize delays in data transmission between nodes in the network, network balancing and avoiding network traffic and overhead, save energy, and maximize the network lifetime. The algorithms that will be covered in this paper are ant colony optimization (ACO), particle swarm optimization (PSO), and artificial bee colony (ABC) by going deeply in describing the criteria of their work and analyses their models.

Keywords: WSN, SI-Based Optimization Algorithms, Ant Colony Optimization (ACO), Particle Swarm Optimization (PSO), Artificial Bee Colony (ABC)
\end{abstract}

\section{INTRODUCTION}

The need to enhance WSN abilities becomes inevitable due to the limited source of energy of each sensor node. Previous research has shown how successful is the artificial intelligent system in achieving the highest level of developments in WSN specifically swarm intelligence based on optimization algorithms, Swarm intelligence is the system that deals with natural and artificial systems containing several individuals that cooperate using decentralized control and self-organization. However, the system focuses on the mutual behaviors that came from the local communication and interaction between the individuals and them with their environment. Examples of systems use swarm intelligence are ant colonies, bird flocking, bacterial growth, fish schooling, microbial intelligence, and swarm robotics. Some human behaviors also can be considered as a part used by swarm intelligence.
Nowadays, most proposed routing protocols is improved by using intelligent systems especially hierarchal routing protocols such as LEACH, PEGASIS, TEEN, APTEEN, HEED and a lot of other cluster-based routing protocols with the most popular swarm intelligence optimization system such as (ACO) ant colony optimization system, (PSO) particle swarm optimizer, (ABC) bee colony algorithm and many other algorithms for optimization [1-2$3]$. The paper is organized as follows. In section 1, that has the biggest part of the paper which offering basic details about popular and specific types of swarm intelligence optimization with going deeply in describing their mechanism and analysis their models. In section 2 summarize the difference between the specific three SIbased optimization algorithms by making a comparison between their merits and demerits also by mention the application that is applied and used by it. Finally, section 3 concludes the paper.

\section{SWARM OPTIMIZATION SYSTEM}

This section will offer basic details about specific types of SI-based optimization algorithms, thesedetails are contenting a description of theworking mechanism of each algorithm by highlighting their Observed variables and their models.

A. Ant Colony Optimization Algorithm (ACO): ACO is the most popular swarm intelligence algorithm that is used to solve optimization problems depending on the ants' real behavior. It is a metaheuristic approach inspired by the Ant System (AS) proposed by Marco Dorigo in 1992 in his Ph.D. thesis [4]. The colonies of ants always Work in an organized manner thanks to their accurate, exact, precise mechanism that is based on the trails which initiate by other previous ants pheromones during traveling in the search space, pheromones are chemicals split by ants to make Tracking process easier for other ants. The goal of ants is to find the best path between the nest and the food, for this reason, the nature of exploration in ants take it is a position to go forward and discover new paths, any paths that have higher amounts of pheromones will be the candidate to consider as the best path. In other words, the probability of selecting ants for the path depends on the pheromones level. So, the intensity of the trail can be considered as a global memory of the system. 

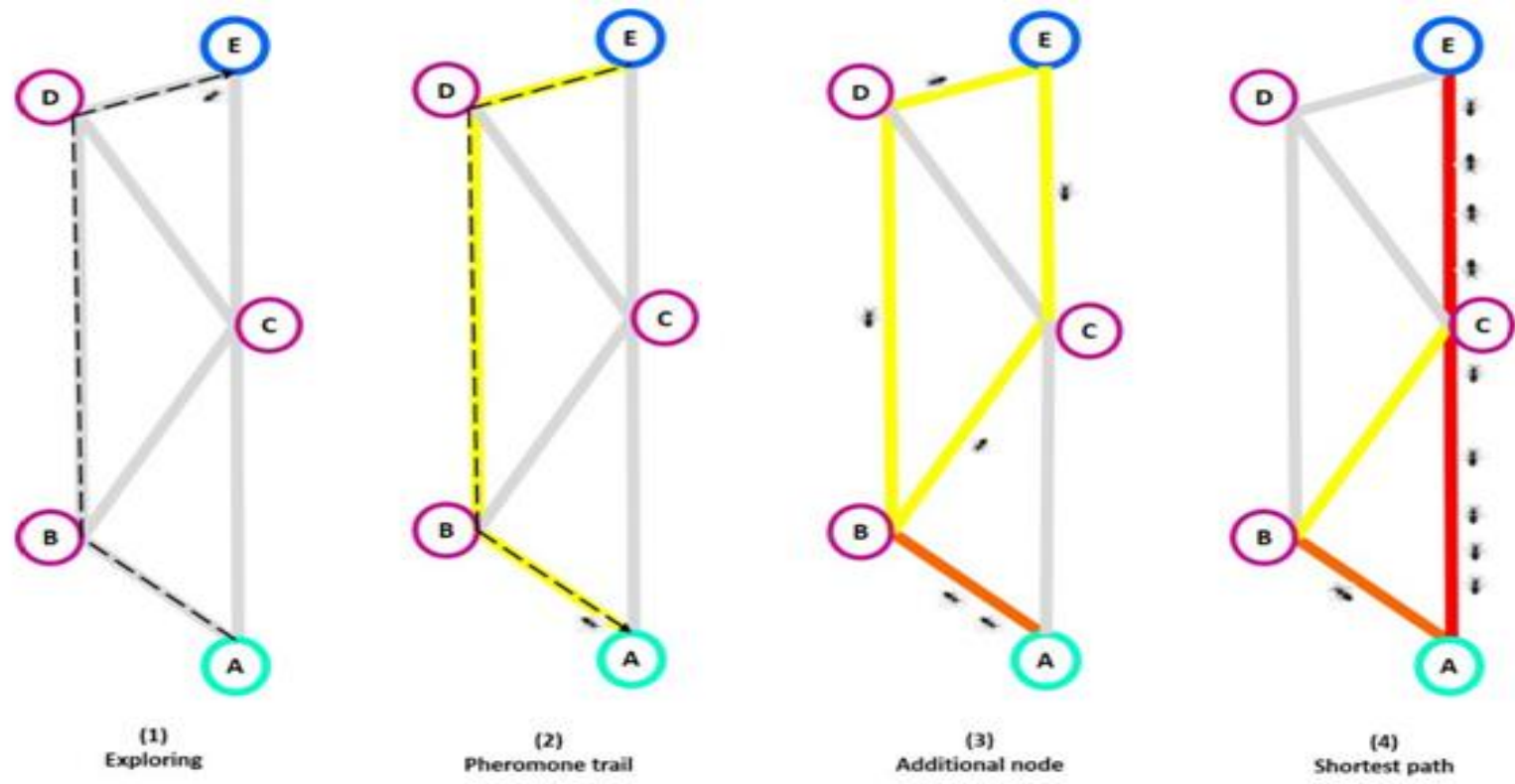

Fig. 1 basic ACO behavior

The previous figure shows the initial steps of ants algorithms to find the best path between the nest and the food, in this case considering $\mathrm{A}$ is the nest and $\mathrm{E}$ is the food the rest of points is middle stations $(\mathrm{B}, \mathrm{C}, \mathrm{D})$. Thefirst step of ants algorithm is discovering the searching area by sending one ant to discover the area, find the food, and comeback to the nest during this process the ant is Dissemination it is pheromones that will create the pheromone trail, the rest of ants will consider the path in yellow color (A-B-D-E) is the best path. After a short time, the distance from point $A$ to point $B$ is being more attractive to ants, in the figure this attractive path turns to orange color to show Increasing importance of this path for other ants. So, during the time another ant is going forwardalone to different directionstarting from the new point $\mathrm{B}$ to discover another shortest path that will be shorter than the previous one. This process is continued until finding the shortest path that appears in red color (A-C-E). The following equation represents the previous process mathematically [4-5]:

$$
P_{(i, j)}^{k}(t)=\frac{\left(\left[\tau_{i j}(t)\right]^{\alpha} \cdot\left[\eta_{i j}\right]^{\beta}\right)}{\left(\sum_{k \in J_{k}}\left[\tau_{i j}(t)\right]^{\alpha} \cdot\left[\eta_{i j}\right]^{\beta}\right)}
$$

1. $P_{(i, j)}$ : The probability of traveling from node $\mathrm{i}$ to node $\mathrm{j}$. 2. $J_{k}$ : The nodes that the antPass through it and travel to from node i.

3. $\eta_{i j}$ : Contributes to the visibility between node $\mathrm{i}$ and node $\mathrm{j}$.

4. $\tau_{i j}(t)$ : Shows the amount of un-evaporated pheromone between node $i$ and node $j$ at time $t$.

5. $\alpha$ and $\beta$ : Control the effect of $\tau i j(t)$ and $\eta \mathrm{i} j$.

6. Where if $\alpha>\beta$ then the searching behavior of ant is more depending on the pheromone.
7. And if $\beta>\alpha$ then the searching behavior of ant is depending on its visibility or knowledge.

In addition, each ant has a taboo list that is used to ban any ants from going to the same node more than one time. As previously mentioned pheromones are one of the primary components in $\mathrm{ACO}$ as they produce trails that maximize the probability of the next ant choosing the same path. The following equation is used to express the process of depositing a pheromone [4-5]:

$$
\Delta \tau_{i j}^{k}(t)=\left\{\begin{array}{c}
\frac{Q}{L_{k}}(t) \\
0
\end{array}(2)\right.
$$

1. Q: is a constant.

2. L: is the cost of the ant's tour, (i.e., the length of the generated path).

3. $\mathrm{t}$ : is the iteration number.

4. K: represents the number of ants.

5. $\Delta \tau_{i j}^{k}(t)$ : Represents the pheromone rate between node $\mathrm{i}$ and node $\mathrm{j}$ that the ant visited in iteration $\mathrm{t}$.

6. The pheromone deposition value for a path that is not selected is zero.

The other important issue is the pheromone evaporation rate, too low values result in disability to find the optimal path whereas too high exploration rates result in ants getting lost, [4-5-6]. Thefollowing equation is using to utilize the pheromone decay factor:

$$
\tau_{i j}(t+1)=(1-p) \cdot \tau_{(i, j)(t)}+\sum_{(k=1)}^{m}\left[\Delta \tau_{(i, j)}^{k}(t)\right](3)
$$

7. m: represents the number of ants in the system.

8. $\mathrm{p}:$ represents the pheromone evaporation rate or decay factor. 


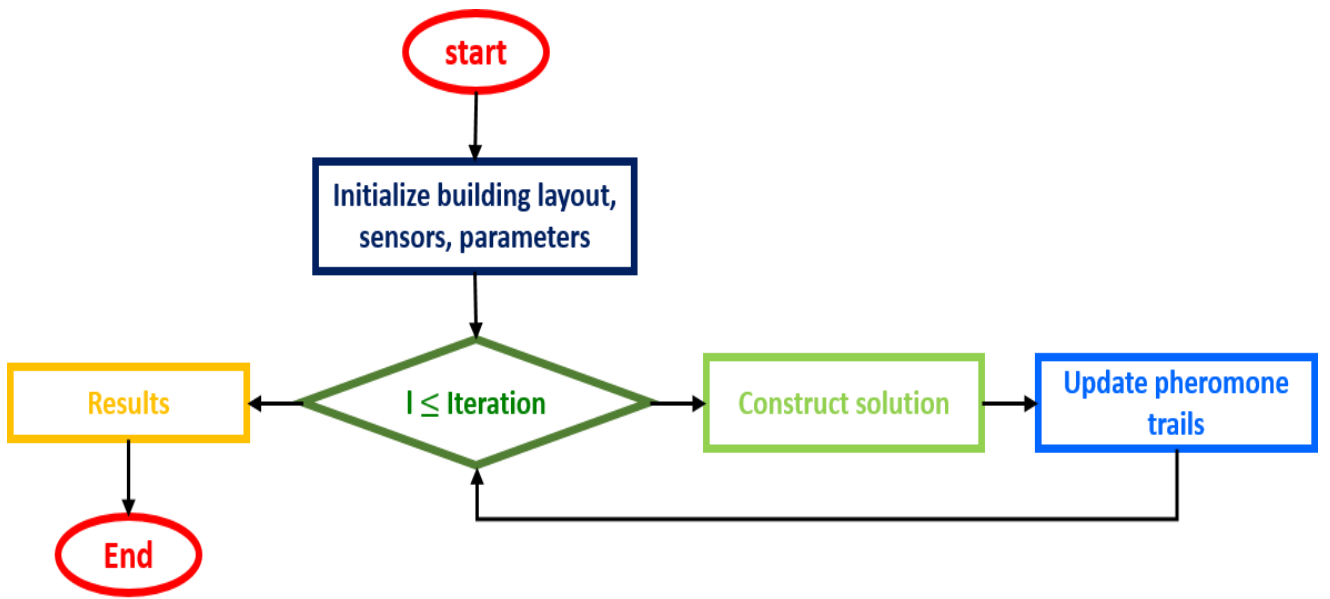

Fig. 2 Flowchart of ACO Applied to WSNs

B. Particle Swarm Optimization Algorithm:Particle Swarm Optimization (PSO) is an optimization strategy represented by Kennedy and Eberhart in 1995 [7]. It uses a simple criterion that mimics swarm behavior in birds flocking and fish schooling to guide the particles to search for global optimal solutions due to different problem existence. Del Valle and his co-authors [8] described PSO in a quick review by partitionPSO process to three simple behaviors of separation, alignment, and cohesion as shown in fig 3. Fig. 3.1 shows separation behavior where particle avoiding other particles, fig.3.2 shows alignment behavior where particle moving towards head of local flockmates and maintain the speed between them, and figure 3.3 shows cohesion behavior where particle moving towards the average position of local flockmates.

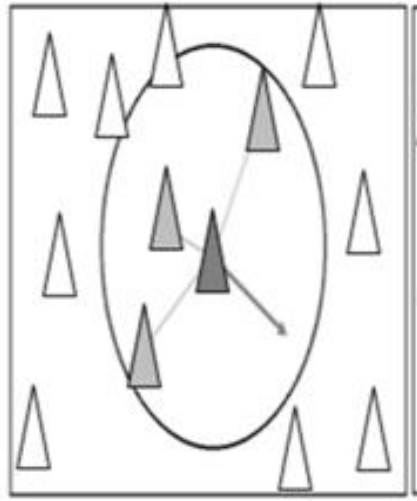

1

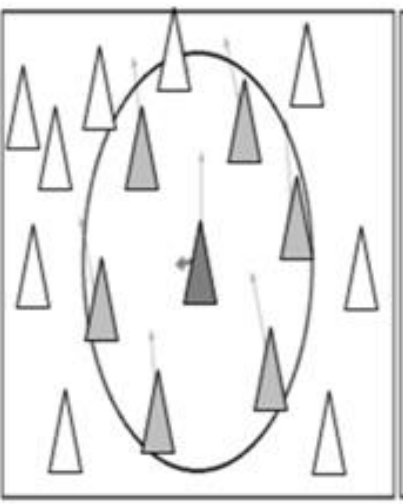

2

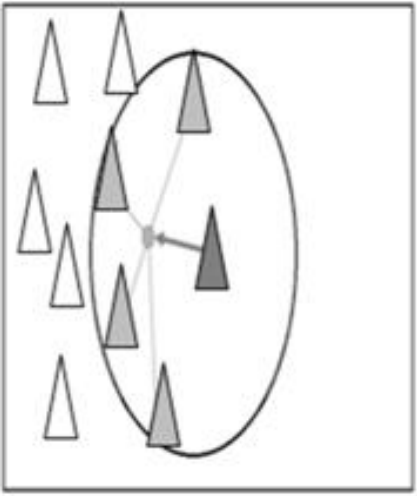

3

Fig. 3 Basic PSO Behavior

The formulas of the PSO algorithm are as follows [7]:

$v_{i d}^{t+1}=v_{i d}^{t}+c_{1} \cdot \operatorname{rand}(0,1) \cdot\left(p_{i d}^{t}-x_{i d}^{t}\right)+$

$c_{2} \cdot \operatorname{rand}(0,1) \cdot\left(p_{g d}^{t}-x_{i d}^{t}\right)(4)$

$$
x_{i d}^{t+1}=x_{i d}^{t}+v_{i d}^{t+1}
$$

1. $v_{i d}^{t}$ : particle velocity

2. $x_{i d}^{t}$ : particle position

3. $d$ : is the dimension in the search space

4. $i:$ is the particle index

5. $t:$ is the iteration number.

6. $c_{1}$ and $c_{2}$ : represent the speed, regulating the length when flying towards the most optimal particles of the whole swarm and the most optimal individual particle.
7. $p_{i}:$ is the best position achieved so far by particle $\mathrm{i}$ and pg is the best position found by the neighbors of particle $i$.

8. $\operatorname{rand}(0,1)$ : are the random values between 0 and 1 .

9. The exploration happens if either or both of the differences between the particle's best $\left(p_{i d}^{t}\right)$ and previous particle's position ( $\left.x_{i d}^{t}\right)$, and between population's all-time best $\left(p_{g d}^{t}\right)$ and previous particle's position $\left(x_{i d}^{t}\right)$ are large, and exploitation occurs when these values are both small. 


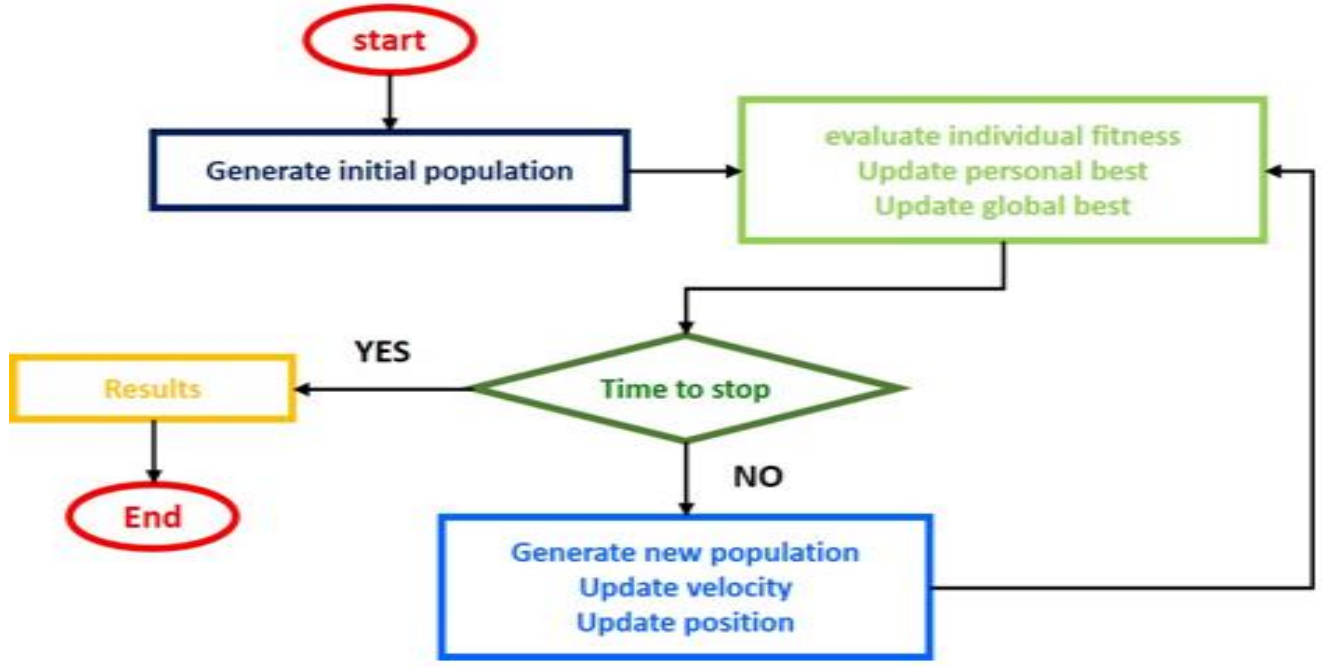

Fig. 4 Flowchart of PSO Applied to the WSNs

The PSO algorithm begins by initializing the population first. The second step is calculating and evaluates individual fitness values of each particle, then updating personal and global bests, finally, updating the velocity and the position of the particles. The second to final steps get repeated until the termination condition is satisfied [8-9]. Fig 5 illustrates the PSO algorithm solution. Each particle is evaluated. The best solutions are found with respect to neighborhood topology and the personal and global best particles for each member of the swarm are updated. The convergence would be achieved by attracting all particles towards the particle with the best solution.A movement towards global optima over iteration numbers [9-10].
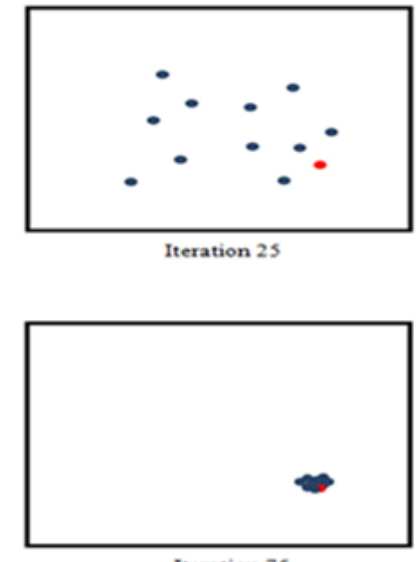

Iteration 75

Iteration 50

Fig. 5 Particle Swarm Optimization

C. Artificial Bee Colony: Artificial Bee Colony (ABC) is one of the most recent swarm intelligence algorithms. It was proposed by DervisKaraboga in 2005 [11]. This algorithm is inspired by the intelligent behavior of real honey bees in finding food sources, known as nectar, and the sharing of information about that food source among other bees in the nest.

This algorithm is claimed to be as simple and easy to implement as PSO. In this approach, Artificial agents are defined and categorized into three types:

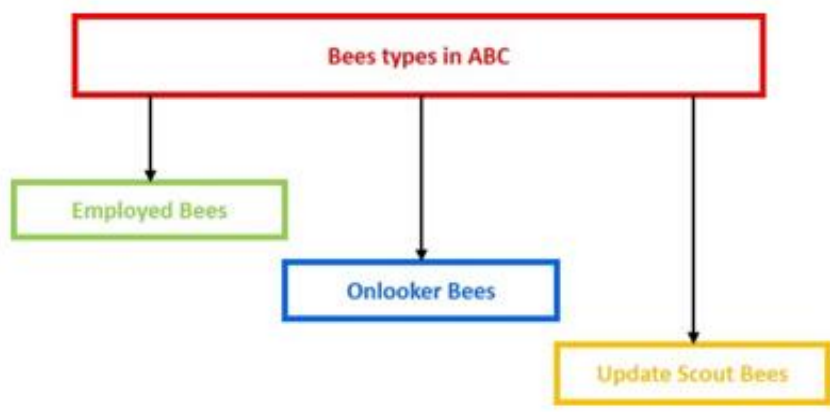

Fig. 6 Types of Bees in ABC 
Each of these bees has different missions allocated to them in order to complete the algorithm's process. The employed bees are responsible for searching food around a food source and save in their memories these food sources. At the other hand, each food source needs in returnone bee to be responsible for this specific food source. So, the number of employed bees is equal to the number of food sources. The onlooker bee is responsible for gathering the nectar from the best food source selection based on the information received from the employed bees in the hive. The scout bee is responsible for search and explores a new destination and new food resources. The general process of the $A B C$ method and the details of each step are as follows [11]:

1. Levell. Initialization Phase: The following equation is used for the initialization phase were food sources is generated randomly:

$$
x_{i}=l_{i}+\operatorname{rand}(0,1) *\left(u_{i}-l_{i}\right)(6)
$$

a) $l_{i} \& u_{i}$ : present respectively the lower and upper bound parameters of xi.

2. Level 2. Employed Bees Phase: In this phase, the search for a new food source $\left(\overrightarrow{v_{l}}\right)$ increases in order to have more nectar around the neighborhood of the current food source $\left(\overrightarrow{x_{l}}\right)$. Once a neighboring food source is located and selected, its fitness is evaluated for each employed bees. Each new neighboring food source will have the following equation:

$$
v_{i}=x_{i}+\Phi !\left(x_{i}-x_{j}\right)(7)
$$

a) $x_{i}$ : is a food source which selected randomly.

b) $\Phi !$ is a random number of $[-\mathrm{g}, \mathrm{g}]$.

c) Once the new food source is found, $v_{i}$ produced its profitability by measuring and applying greedy selection between $x_{i}$ and $v_{i}$.

d) The exploration begins if the difference between $x_{i}, x_{j}$ is large.

e) The exploitation behavior stops when the difference between $x_{i}, x_{j}$ is small.

The fitness value of the solution $\mathrm{fit}_{i}\left(\overrightarrow{x_{i}}\right)$ is determined using the following equation:

$$
\text { fit }_{i}\left(\overrightarrow{x_{i}}\right)=\left\{\begin{array}{c}
\frac{1}{1+f_{i}\left(\overrightarrow{x_{i}}\right)} \text { if } f_{i}\left(\overrightarrow{x_{i}}\right) \geq 0 \\
1+a b s\left(f_{i}\left(\overrightarrow{x_{i}}\right)\right) \text { if } f_{i}\left(\overrightarrow{x_{i}}\right)<0
\end{array}\right.
$$

Where $f_{i}\left(\overrightarrow{x_{i}}\right)$ is the objective function value of the solution $\overrightarrow{x_{i}}$.

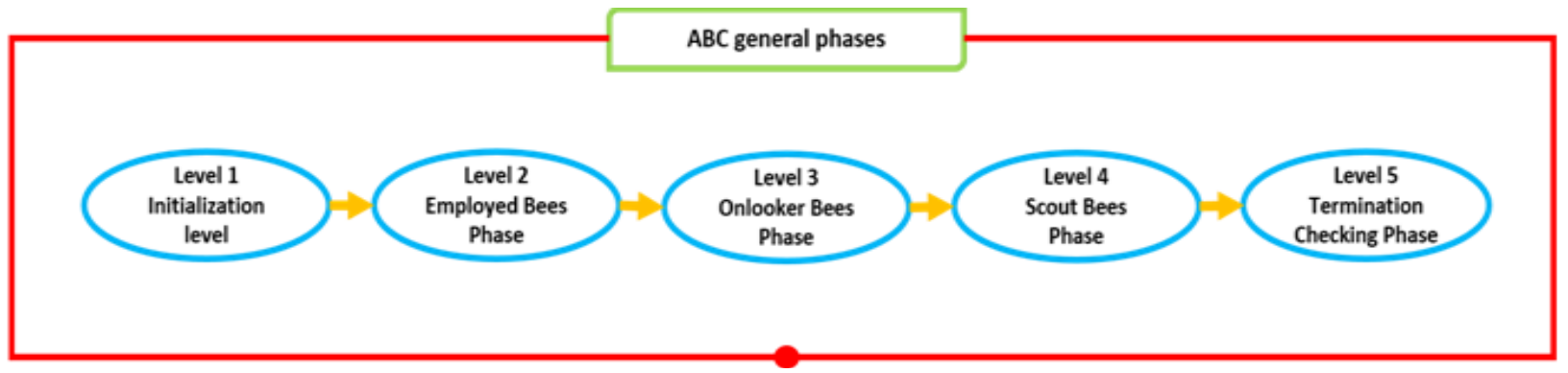

Fig. 7 ABC General Phases Algorithm

3. Level 3. Onlooker Bees Phase:In meanwhile Onlooker bees are located in the hive waiting for the fitness value and the information shared by employed bees and measures the probability values to choose their food sources. The probability value $p_{i}$ is representing in the following equation:

$$
p_{i}=\frac{\text { fit }_{i}\left(\overrightarrow{x_{i}}\right)}{\sum_{\mathrm{i}=1}^{S N} f i t_{i}\left(\overrightarrow{x_{i}}\right)}(9)
$$

4. Level 4. Scout Bees Phase:The scout bees are those unemployed bees that choose their food sources randomly. Employed bees which cannot improve their fitness value through a Predefinednumber of iteration they became as scout bees and all their food sources get abandoned, called as limit or abandonment criteria. While the employed bees that have the best fitness value will initiate a position associated with that value to be memorized.

5. Level 5. Termination Checking Phase: If the termination condition is happened by giving the wanted results, the program terminates, otherwise, the program returns to level 2 and repeats until the termination condition is met [11].

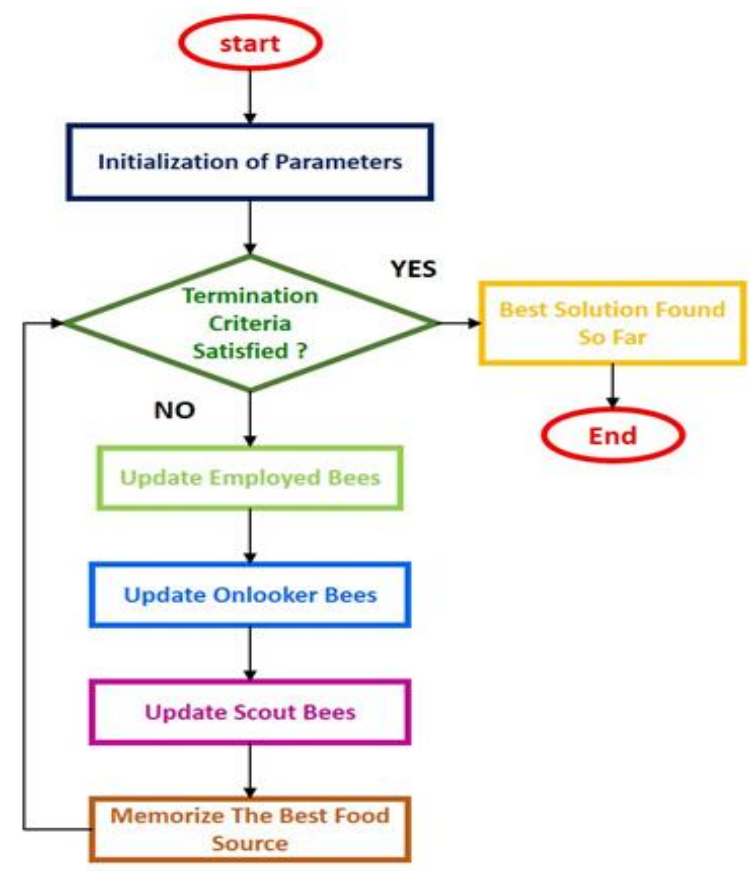

Fig. 8 Flowchart of ABC Applied to the WSNs 


\section{COMPARISON}

This section summarizes the difference between the specific three SI-based optimization algorithms by mention their merits and demerits.

TABLE I ACO - PSO - ABC COMPARISON

\begin{tabular}{|c|c|c|c|}
\hline $\begin{array}{c}\text { Swarm } \\
\text { intelligence } \\
\text { algorithms }\end{array}$ & Merits & Demerits & Applications \\
\hline $\begin{array}{c}\mathrm{ACO} \\
{[12-13-14]}\end{array}$ & $\begin{array}{l}\text { 1. Mainly applied to find a solution for } \\
\text { traveling salesman problem (TSP). } \\
\text { 2. It has proven high performance to deal } \\
\text { with similar NP-hard problems such } \\
\text { as: } \\
\text { - Assignment problem. } \\
\text { - Schedule generation problem. } \\
\text { - Bin-packing problems. }\end{array}$ & $\begin{array}{l}\text { 1. Slower convergence } \\
\text { compared with other } \\
\text { heuristic-based methods and } \\
\text { lacks a centralized processor } \\
\text { to guide it towards good } \\
\text { solutions. } \\
\text { 2. Poor performance within } \\
\text { problems with large search } \\
\text { spaces. }\end{array}$ & $\begin{array}{l}\text { ACO has been applied in various } \\
\text { optimization problems such as: } \\
\text { 1. Traveling salesman problem } \\
\text { (TSP). } \\
\text { 2. Quadratic assignment problem. } \\
\text { 3. Vehicle routing network model } \\
\text { problem. } \\
\text { 4. Image processing. } \\
\text { 5. Path planning for a mobile } \\
\text { robot. } \\
\text { 6. Path optimization for UAV } \\
\text { 7. System. }\end{array}$ \\
\hline $\begin{array}{c}\text { PSO } \\
{[15-16-17]}\end{array}$ & $\begin{array}{l}\text { 1. Simple to implement. } \\
\text { 2. Has only a few parameters to be set. } \\
\text { 3. It is effective in a global search. } \\
\text { 4. It is insensitive to scaling of design } \\
\text { variables. } \\
\text { 5. It is easily parallelized for concurrent } \\
\text { processing. }\end{array}$ & $\begin{array}{l}\text { 1. Has a tendency to result in a } \\
\text { fast and premature } \\
\text { convergence in mid optimum } \\
\text { points. }\end{array}$ & $\begin{array}{l}\text { PSO is used in: } \\
\text { 1. Networking. } \\
\text { 2. Power systems. } \\
\text { 3. } \quad \text { Signal processing. } \\
\text { 4. } \quad \text { Control system. } \\
\text { 5. Machine learning. } \\
\text { 6. } \quad \text { Image processing. } \\
\end{array}$ \\
\hline $\begin{array}{c}\mathrm{ABC} \\
{[18-19-20]}\end{array}$ & $\begin{array}{l}\text { 1. easy to implement, } \\
\text { 2. Robust and highly flexible. } \\
\text { 3. It is considered as highly flexible since } \\
\text { only requires two control parameters } \\
\text { of maximum cycle number } \\
\text { and colony size. } \\
\text { 4. Adding and removing a bee can be } \\
\text { done without the need to reinitialize } \\
\text { the algorithm. } \\
\text { 5. It can be used in many optimization } \\
\text { problems without any modification. } \\
\text { 6equires fewer control parameters } \\
\text { compared with other search } \\
\text { techniques. }\end{array}$ & $\begin{array}{l}\text { 1. The requirement of new } \\
\text { fitness tests for the new } \\
\text { parameters to improve } \\
\text { performance. } \\
\text { 2. Being quite slow when used } \\
\text { in serial processing, and the } \\
\text { need for a high } \\
\text { amount of objective function } \\
\text { evaluations. }\end{array}$ & $\begin{array}{l}\text { ABC has been implemented in } \\
\text { various fields including: } \\
\text { 1. Engineering design problems } \\
\text { 2. Networking. } \\
\text { 3. Business. } \\
\text { 4. Electronics. } \\
\text { 5. Scheduling. } \\
\text { 6. Image processing. }\end{array}$ \\
\hline
\end{tabular}

\section{CONCLUSION}

The main aim of this paper is to give a review with deep details about the most popular and used Swarm Intelligence (SI) algorithmsincluding Ant Colony Optimization (ACO), Particle Swarm Optimization (PSO), Artificial Bee Colony (ABC). Where have been described previously in more than one issue due to their pivotal role in every field and study especially networking and WSN routing protocols to be enhanced and improved. This paper presents each of these three swarm optimization algorithms by describing their work mechanism, show their models, and summarizes them by showing a brief comparison between their merits and demerits with the most important applications.

\section{REFERENCES}

[1] J. N. Al-Karaki and A. E. Kamal "Routing Techniques in Wireless Sensor Networks: A Survey", IEEE-Security and Networks, Vol. 11, No. 6, pp. 6-28, 2004.
[2] S. Misra, and S. Goswami, "Network Routing: Fundamentals, Applications, and Emerging Technologies", 1st ed, John Wiley \& Sons Ltd., Ch.11, pp. 285-325, 2017.

[3] S. K. Singh, M. P. Singh, and D. K. Singh, "Routing Protocols in Wireless Sensor Networks - A Survey", International Journal of Computer Science \& Engineering Survey (IJCSES), Vol. 1, No. 2, pp. 63-83,2010.

[4] M. Dorigo, "Optimization, learning and natural algorithms", Ph.D. Thesis, Politecnico di Milano, Milan.1992. [Online] Available: http://ci.nii.ac.jp/naid/10016599043/

[5] M. Dorigo, M.Birattari, and T. Stutzle, "Ant Colony Optimization. Computational Intelligence Magazine", IEEE, pp. 28-39,2006

[6] Y. Pei, W. Wang, and S. Zhang, "Basic Ant Colony Optimization". International Conference on Computer Science and Electronics Engineering, pp. 665-667, 2012.

[7] J. Kennedy, and R.Eberhart, "Particle swarm optimization". IEEE International Conference on Neural Networks, pp. 1942-1948, 1995.

[8] Y. Del Valle, G. K. Venayagamoorthy, S.Mohagheghi, J. C. Hernandez, and R. G. Harley, "Particle Swarm Optimization: Basic Concepts, Variants, and Applications in Power System", IEEE Trans Evolutionary Computer, pp. 171-195, 2008. 
[9] A. Banks, J. Vincent, and C. Anyakoha, "A Review of Particle Swarm Optimization. Part I: Background andDevelopment", Springer Science, pp. 467-484, 2007.

[10] S. Jiang, "LEACH Protocol Analysis and Optimization of Wireless Sensor Networks Based on PSO and AC", IEEE, Proceedings of 10th International Conference on Intelligent Human-Machine Systems and Cybernetics, 978-1-5386-5836-9/18, pp. 246-250, 2018.

[11] D. Karaboga, "An idea based on honeybee swarm for numeric optimization”, Technical Report TR06, Erciyes University, 2005.

[12] V. Selvi, and R. Umarani, "Comparative Analysis of Ant Colony and Particle Swarm Optimization Techniques", International Journal of Computer Applications, pp. 1-6, 2010.

[13] B. Yang, Q. Ma, and J. Wang, "Study on the Routing Technology of Wireless Sensor Network Based on Ant Colony Optimization", Journal of Sensor Technology, Vol. 6, pp. 141-158, 2016.

[14] A. Nayyar, "A Comprehensive Review of Ant Colony Optimization (ACO) based Energy-Efficient Routing Protocols for Wireless Sensor Networks", International Journal of Wireless Networks and Broadband Technologies, Vol. 3, No. 3, pp. 33-55, 2014.
[15] Q. Bai, "Analysis of Particle Swarm Optimization Algorithm", Computer and Information Science, pp.180-184, 2010

[16] M. Gong, Q. Cai, X. Chen, and L. Ma, "Complex Network Clustering by Multi-objective Discrete ParticleSwarm Optimization Based on Decomposition", IEEE Transaction on Evolutionary Computation, Vol. 18, No.1, pp. 82-97, 2014.

[17] F. H. Ali, and H. N. Abdulrazzak, "Pattern Recognition Based On Intelligent System", Al-Rafidain University College For Sciences, Vol. 31, pp. 57-77, 2013

[18] F. S. Abu-Mouti, and M. E. El-Hawary, "Overview of Artificial Bee Colony (ABC) algorithm and its applications", International Systems Conference-SysCon, pp. 1-6, 2012.

[19] E. Gerhardt, and H. M. Gomes, "Artificial Bee Colony (ABC) Algorithm for Engineering Optimization Problems", International Conference on Engineering Optimization, pp.1-11, 2012.

[20] D. Karaboga, B. Gorkemli, C. Ozturk, and N. Karaboga, "A comprehensive survey: artificial bee colony (ABC) algorithm and applications", Artificial Intelligence Review, Vol. 42, No.1, pp. 21-57, 2014. 